\title{
Structural transition of carbon nanoparticles caused by energetic collisions
}

\author{
Ri Cai, Jun Ye, Qingbin Wang, Guohui Chen, Junting Liu, Liangyuan Shi, Mingwei Lin, \\ Zhuoming Xie, Jianfu Liang, Hui Huang, Jinming Liu and Mingjun Li \\ Yunfu Power Supply Bureau of Guangdong Power Grid Co., Ltd., \\ Yunfu 527300, China \\ Cheng Liu* \\ Department of Vascular Surgery, Nanjing Drum Tower Hospital, \\ The Affiliated Hospital of Nanjing University Medical School, \\ Nanjing 210093, China \\ dida6666@163.com \\ Hanbiao Song* \\ State Grid Liaoyang Power Supply Co., Ltd., \\ Liaoyang 111000, China \\ 13704990678@163.com
}

Received 21 August 2017

Revised 11 September 2017

Accepted 15 September 2017

Published 28 September 2017

\begin{abstract}
A broad general interest in the process of affecting the energetic deposition of flying nanoparticles has prompted a new opportunity to consider the medium-energy region in which the nanoparticles maintain their integrity and must respond to the energetic shock caused by collisions. We herein report our experimental results on the structural transition of the carbon nanoparticles induced by such energetic collisions. The beam energy was varied from $0 \mathrm{keV}$ to $30 \mathrm{keV}$ for a nanoparticle with over 5000 carbon atoms. Electron energy loss spectroscopy, X-ray photoemission spectroscopy, and Raman spectroscopy were carried out on the samples using a series of beam energies. These tests revealed that some graphitic sheets were formed at the higher beam energy while the $s p^{3}$ ratio was unexpectedly higher for a medium beam energy.
\end{abstract}

Keywords: Carbon nanoparticles; structural transition; electron energy loss spectroscopy; energetic collisions.

* Corresponding authors.

This is an Open Access article published by World Scientific Publishing Company. It is distributed under the terms of the Creative Commons Attribution 4.0 (CC-BY) License. Further distribution of this work is permitted, provided the original work is properly cited. 


\section{R. Cai et al.}

\section{Introduction}

The process of collision of flying nanoparticles at a solid surface is of broad physical interest. ${ }^{1-4}$ The kind of change that takes place in these nanoparticles when they collide with a solid surface energetically has been considered in different cases, as specified by the average kinetic energy per atom in the nanoparticles. ${ }^{1-4}$ The average kinetic energies $\left(E_{k}\right)$ are comparable with the average atomistic binding energies because the inner bonding is the critical difference between a nanoparticle and a loosely-packed group of the same atoms. $\frac{5,6}{}$ The nanoparticles land softly with no obvious structural change when their kinetic energy is much lower than the binding energies $\left(E_{k}<0.1 \mathrm{eV} /\right.$ atom). Molecular dynamical simulations indicate that the nanoparticles are crushed completely when their kinetic energies are much greater than the binding energies $\left(E_{k}>10 \mathrm{eV} /\right.$ atom $) .11$ A study of this type also provides a good opportunity to prepare atomically smooth films, as demonstrated experimentally. ${ }^{1-11}$ There is still no clear consensus on how nanoparticles behave when they collide at a medium kinetic energy comparable to the binding energy $\left(E_{k} \sim 1 \mathrm{eV} /\right.$ atom $)$, although some theoretical approaches have been proposed. $12-14$ It is reasonable to assume that the nanoparticles will not be crushed by the energetic collision, but instead be pressed against the interatomic potential inside the deposited nanoparticles. This may allow reconstruction within the nanoparticles at the atomic level, providing an opportunity to generate the structural transition of the nanoparticles by energetic collision with a medium kinetic energy. We report here on the experimental evidence of the occurrence of such structural transitions.

\section{Experimental Procedure}

Carbon nanoparticles were selected in this experiment in view of their significance in the graphite-diamond transformation, in the synthesis of nanosized diamonds, and in the establishment of the size-related phase diagram of nanocarbon. $\frac{15-18}{-18}$ The experiments were carried out using a home-made instrument with a gas-aggregation nanoparticle source and a group of planar electrostatic electrodes. The nanoparticles were generated by the source, and carbon nanoparticles with a diameter of 2-3 nm were selected to form a particle beam. The nanoparticles were then accelerated causing collision with substrates (Formvar and silicon) at kinetic energies from a few eV up to $30 \mathrm{keV}$, corresponding to a directional flight velocity of $10^{2}-10^{4} \mathrm{~m} / \mathrm{s}$. The $s p^{3}$-hybridized atom ratio (the $s p^{3}$ ratio) in the carbon nanoparticles is usually used to describe their structures and determine their physical performance, i.e. how diamond-like they are. $\frac{18}{}$ This $s p^{3}$ ratio was characterized by means of electron energy-loss spectroscopy (EELS) attached in a transmission electron microscopy (TEM). The change in the $s p^{3}$ ratio with respect to the change in the kinetic energy of the incident nanoparticles was therefore analyzed by considering three categories, i.e. no obvious change, becoming more diamond-like, and becoming more graphitelike, respectively. 


\section{Results and Discussion}

A transition towards more graphite-like structures is clear in our results. Figure 1(a) shows the typical EELS curves from samples with beam kinetic energies of 0,10 , 15,20 , and $30 \mathrm{keV}$. The nanoparticles contain around 5000 atoms as determined by the detailed analysis of scanning TEM results. The EELS spectra show that the diamond-like bonding dominates in the nanoparticles with kinetic energies in the range of $0-15 \mathrm{keV}(\sim<3 \mathrm{eV} /$ atom $) .{ }^{19}$ A sharp $\pi$ peak located in the energy range of $280-290 \mathrm{eV}$ is apparent, indicating that the dominance of $\pi$ bonding after the nanoparticles has collided with the surface at a kinetic energy of $20 \mathrm{keV}$ (> $4 \mathrm{eV} /$ atom).

The detailed analysis shows the results in terms of the kinetic energy-dependent structures of the nanoparticles, as shown in Fig. 1(b), in which the $s p^{3}$ ratio is plotted against the kinetic energy of the flying nanoparticles. $\frac{19}{}$ These data were obtained by fitting the EELS peaks using the standard spectra of graphite and diamond. The estimated velocity is shown in the upper $x$-axis. The $s p^{3}$ ratio of soft-landing nanoparticles is $53 \%$ and the ratio at a kinetic energy of $5 \mathrm{keV}$ is $56 \%$. This implies no obvious change in structure. The $s p^{3}$ ratio decreases to $20 \%$ when the kinetic energy increases to $20 \mathrm{keV}$ and then decreases to $17 \%$ at $25 \mathrm{keV}$ and $8 \%$ at $30 \mathrm{keV}$. The nanoparticles are in fact almost totally graphitized, confirming the structural transition towards graphite. It is interesting to note that the $s p^{3}$ ratio increases steadily to $85 \%$ as the kinetic energy increases from $5 \mathrm{keV}$ to $15 \mathrm{keV}$, which indicates the transition of the structures in a more diamond-like direction.

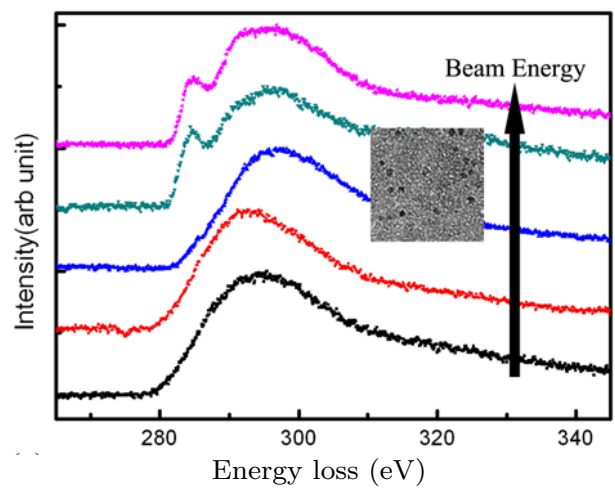

(a)

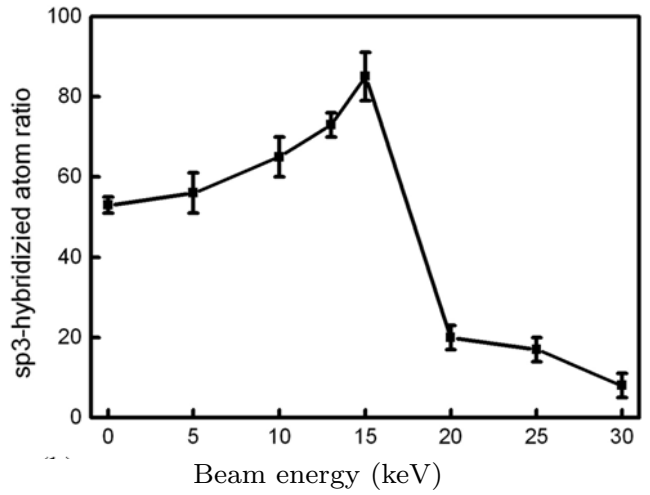

(b)

Fig. 1. EELS analysis of the deposited carbon nanoparticles on Formvar films with various energies. (a) EELS data for the core loss of the carbon edge for the low-coverage isolated nano-islands formed by nanoparticles colliding with a range of kinetic energies. The kinetic energies increase from the bottom of the figure upwards. The appearance of the sharp peak in the $280-290 \mathrm{eV}$ energy window marks the formation of graphite-like bonding within the nanoparticles $(\pi$ bond). The inset is a typical TEM image of a sample with the lower beam energy. (b) The ratio of the $s p^{3}$-hybridized atoms plotted against the kinetic energy. The results were obtained from the EELS data using the method used in Ref. 19. The structural transition at a kinetic energy of $20 \mathrm{keV}$ is clearly shown. The upper $x$-axis is the estimated value of the velocity of the nanoparticles. 


\section{R. Cai et al.}

There is evidence that the atoms inside the nanoparticles are reconstructed during the impacts, forming more four-coordinated tetragonal bonds. Notably, a transition towards both more diamond-like and more graphite-like structures is observed.

As stated above, the $s p^{3}$ ratio of the deposited carbon nanoparticles varies with the change in their kinetic energy, which implies the occurrence of the structural transition. Our TEM observation shows that the nanoparticles remain separate after the structural transition (Fig. 1(a)), which implies that each nanoparticle undergoes the transition individually. The carbon nanoparticles are also made to collide energetically with silicon substrates. This is just to exclude the possibility that the structural changes in the nanoparticles occur as a result of a chemical reaction with the substrate materials. The experiments using silicon substrates are carried out simultaneously with the same experiments carried out on Formvar surfaces. The silicon substrates with the deposited nanoparticles are then removed for X-ray Photon-activated electron spectra (XPS) analysis. The XPS results for the $C_{1 s}$ peak are shown in Fig. 2(a). The center of the $C_{1 s}$ peak can be seen to shift slightly towards a higher energy while the beam energy increases from $0 \mathrm{keV}$ to $15 \mathrm{keV}$ before dropping back later on. The spectra are further analyzed according to the previous method. ${ }^{20}$ The relative value of the $s p^{3}$ ratio is plotted against the kinetic energy, as shown in Fig. 2(b). We observe both the transition in a diamondlike direction at a kinetic energy of $5-10 \mathrm{keV}$, and transition in a graphite-like direction at a kinetic energy greater than $15 \mathrm{keV}$. As the incident energy increases, the $s p^{3}$ ratio rises in the lower energy range and falls at the greater incident energy. The trend of the curve is the same as that seen in the experiments with the Formvar surfaces although the point of transition between the rise and fall is different.

The two experiments on Silicon and Formvar show the same results in terms of the kinetic energy-dependent structural transitions of nanoparticles during the energetic collisions on solid surfaces, and reveal the lack of influence of both the

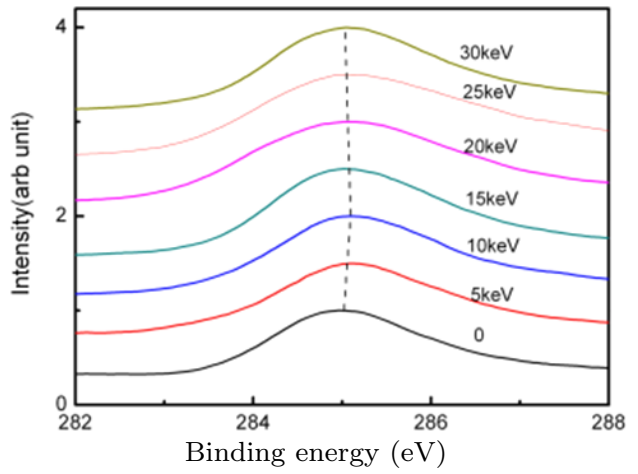

(a)

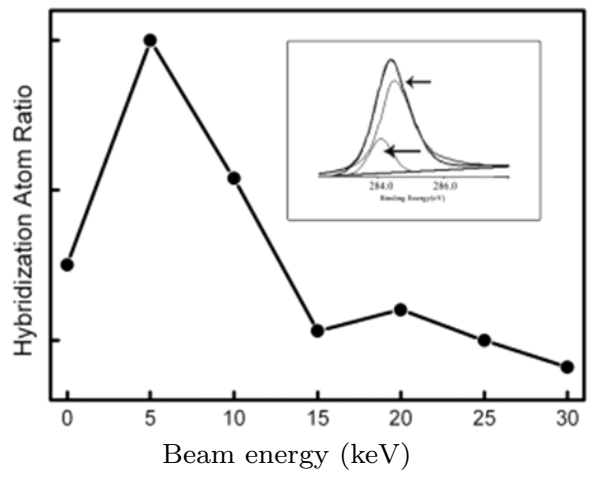

(b)

Fig. 2. XPS analysis of the deposited carbon nanoparticles on silicon surfaces for various energies. (a) The XPS peak of the samples, where the deposition energies are indicated. This is a zoom-in of the $C_{1 s}$ peak, where the shift of the peak is apparent. (b) The ratio of the $s p^{3}$-hybridized atoms plotted against the kinetic energy obtained by fitting the XPS peak as shown in the inset. 
chemical composition of the solid surface and the coalescence of nanoparticles. The observed structural transition must be the result of energetic collisions. In fact, the energy-dependent curves of the structures (Figs. 1(b) and 2(b)) form lines in the size-kinetic energy-structure phase diagram of nanocarbons. The structural changes in the deposited nanoparticles demonstrate that the energy loss effectively influences the carbon nanoparticles. The process is clearly not the same as that when an atomic ion of carbon collides with the surface with the same $E_{k}$ during which most of the impact energy is transferred to the solid surface and penetration is considered to be the dominant effect. The $s p^{3}$ ratio reduces to a very low value at a higher $E_{k}$ and remains low even at the higher energies in both experiments. The same phenomena occur even when $E_{k}$ reaches the high energy region. Therefore, it is reasonable to assume that this loss of $s p^{3}$ may be attributed to the higher energy, essentially in the crushing of the nanoparticles and the resulting loss of the $3 d s p^{3}$ bonding. We highlight the special effect of the increase in the $s p^{3}$ ratio in the medium-energy region before the nanoparticles are crushed. In this region, the particle structures are found to be sensitive to the change of $E_{k}$ as shown in our experiments. It might be expected that the loss of kinetic energy is effectively transferred to the nanoparticles themselves. This deposition of energy affects the interatomic potential in the nanoparticles, favoring the reconstruction of the atoms, and resulting in an energy-dependent structural change.

In Fig. 3, we show the high-resolution TEM images of a sample for a beam energy of $20 \mathrm{keV}$. We note that the nanoparticles maintain their shapes when the beam energy is $15 \mathrm{keV}$, as shown in the inset of Fig. 1(a). However, in Fig. 3(a), the nanoparticles show the obvious coalescence, forming the graphitic sheets. The lattice

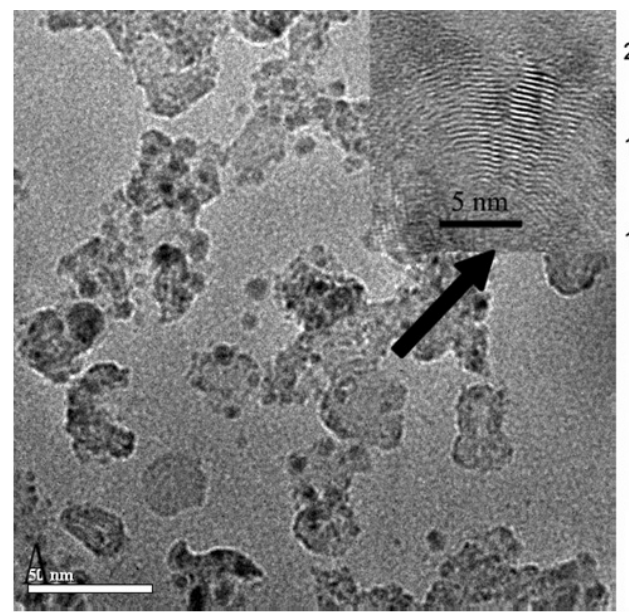

(a)

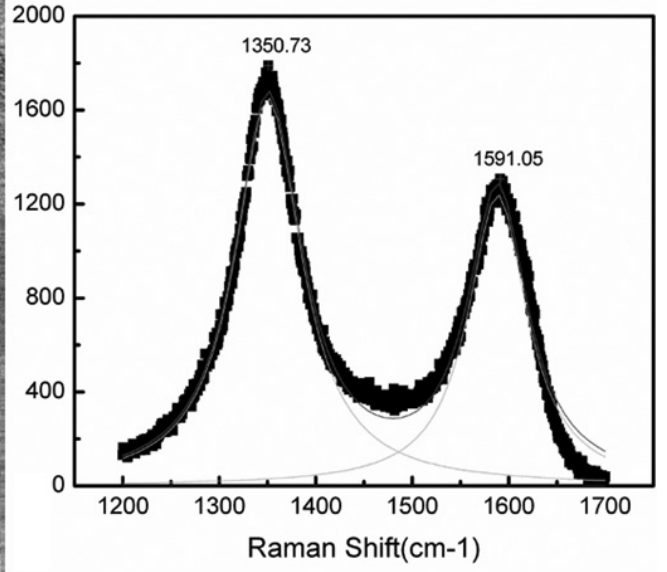

(b)

Fig. 3. Compositional analysis of the samples at the higher beam energy. (a) High resolution TEM image of the samples deposited at $20 \mathrm{keV}$, where some crystalline sheets are formed with the graphite-like wrinkles, as shown in the inset. (b) Raman spectrum of the sample. 
with a spacing of $0.34 \mathrm{~nm}$ can clearly be seen, as shown in the inset. Figure 3(b) shows the Raman spectrum which has two phonon peaks, commonly termed $D$ mode $\left(1350.73 \mathrm{~cm}^{-1}\right)$ and $G$ mode $\left(1591.05 \mathrm{~cm}^{-1}\right)$, respectively, confirming the formation of the disordered graphitic sheets.

\section{Conclusion}

In conclusion, the energetic collision of carbon nanoparticles with a medium average kinetic energy at solid surfaces successfully induces the structural transition of the particles. Transitions in both the diamond- and graphite-like directions then ensue. The increase in the $s p^{3}$ ratio is discovered as a particular effect in the mediumenergy region. These findings may offer a new means of studying phase stability and phase diagrams at the nanoscale.

\section{Acknowledgment}

This work was financially supported by the Science and Technology Project of Guangdong Power Grid Co., Ltd. under Grant No. 035300KK52150004.

\section{References}

1. H. Haberland, Z. Insepov and M. Moseler, Phys. Rev. B 51 (1995) 11061.

2. F. Frost, R. Fechner, B. Ziberi, J. Vollner, D. Flamm and A. Schindler, J. Phys.: Condens. Matter 21 (2009) 224026.

3. M. Castro and R. Cuemo, Appl. Surf. Sci. 258 (2012) 4171.

4. J. Munoz-Garcia, L. Vazquez, M. Castro, R. Gago, A. Redondo-Cubero, A. MorenoBarrado and R. Cuerno, Mater. Sci. Eng. R 86 (2014) 1.

5. R. P. Webb, M. Kerford and A. Way, Nucl. Instrum. Meth. B 153 (1999) 284.

6. I. Yamada, J. Matsuo, Z. Insepov and M. Akizuki, Nucl. Instrum. Meth. B 106 (1995) 165.

7. M. Moseler, P. Gumbsch, C. Casiraghi, A. C. Ferrari and J. Robertson, Science 309 (2005) 1545.

8. T. Ikeda, K. Teii, C. Casiraghi, J. Robertson and A. C. Ferrari, J. Appl. Phys. 104 (2008) 073720.

9. F. Frost, B. Ziberi, A. Schindler and B. Rauschenbach, Appl. Phys. A 91 (2008) 551.

10. S. K. Srivastava, K. Ganesan, P. Gangopadhyay, B. K. Panigrahi, K. G. M. Nair and A. K. Tyagi, Nucl. Instrum. Meth. B 338 (2014) 89.

11. H. Hofsass, K. Zhang and O. Bobes, J. Appl. Phys. 120 (2016) 135308.

12. D. A. Pearson and R. M. Bradley, J. Phys.: Condens. Matter 27 (2015) 015010.

13. Z. C. Yang, M. A. Lively and J. P. Allain, Phys. Rev. B 91 (2015) 075427.

14. G. Hobler, R. M. Bradley and H. M. Urbassek, Phys. Rev. B 93 (2016) 205443.

15. L. Marques et al., Science 283 (1999) 1720.

16. P. Badziag, W. S. Veowoerd, W. P. Ellis and N. R. Greiner, Nature 343 (1990) 244.

17. A. L. Verechshagin, Combust. Explo. Shock Waves 38 (2002) 358.

18. F. Q. Song, X. F. Wang, R. C. Powles, L. B. He, N. A. Marks, S. F. Zhao, J. G. Wan, Z. W. Liu, J. F. Zhou, S. P. Ringer, M. Han and G. H. Wang, Appl. Phys. Lett. 96 (2010) 033103.

19. S. R. P. Silva and V. Stolojan, Thin Solid Films 488 (2005) 283.

20. J. Diaz, G. Paolicelli, S. Ferrer and F. Comin, Phys. Rev. B 54 (1996) 8064. 